\title{
Time evolution in a geometric model of a particle
}

\author{
M.F. Atiyah, ${ }^{a}$ G. Franchetti ${ }^{b}$ and B.J. Schroers ${ }^{b}$ \\ ${ }^{a}$ Maxwell Institute for Mathematical Sciences and \\ School of Mathematics, University of Edinburgh, \\ Kings Buildings, Edinburgh EH9 3JZ, U.K. \\ ${ }^{b}$ Maxwell Institute for Mathematical Sciences and \\ Department of Mathematics, Heriot-Watt University, \\ Riccarton, Edinburgh EH14 4AS, U.K. \\ E-mail: M.Atiyah@ed.ac.uk, g.franchetti@hw.ac.uk, \\ b.j.schroers@hw.ac.uk
}

ABSTRACT: We analyse the properties of a $(4+1)$-dimensional Ricci-flat spacetime which may be viewed as an evolving Taub-NUT geometry, and give exact solutions of the Maxwell and gauged Dirac equation on this background. We interpret these solutions in terms of a geometric model of the electron and its spin, and discuss links between the resulting picture and Dirac's Large Number Hypothesis.

KeYwords: Differential and Algebraic Geometry, Duality in Gauge Field Theories, Solitons Monopoles and Instantons

ARXIV EPRINT: 1412.5915 


\section{Contents}

1 Introduction 1

2 Spin and position in the Taub-NUT geometry 3

2.1 Notation and conventions 3

2.2 Microscopic or spin coordinates 4

2.3 Macroscopic or position coordinates 5

$\begin{array}{ll}2.4 & \text { The twisted Dirac operator on TN space }\end{array}$

2.5 Scaling properties and the Landau limit 8

3 Introducing time $\quad 9$

4 Geometric models of particles and Dirac's Large Number Hypothesis 12

\section{Introduction}

This paper continues the exploration of a purely geometrical description of elementary particles begun in [1] by including time. We focus on one particular model, review its geometry in detail and exhibit a time-dependent version. Our review includes new insights into the description of spin in the geometric framework which build on results obtained in [2].

The work reported here was inspired by Dirac's two-metric formalism, introduced in the context of his Large Number Hypothesis (LNH) [3-6]. The two metrics considered by Dirac - the usual spacetime metric in Einstein's theory of gravitation and a second one for the description of 'atomic' phenomena - are related in a way which is echoed in the relation between the spatial and fibre geometry of the Taub-NUT space. Extending this relation to a spacetime picture led us to the $(4+1)$-dimensional Ricci-flat extension of the Taub-NUT space which we shall discuss, and which had previously been derived in [7].

In the geometric approach of [1], static particles are modelled by four-manifolds, and conserved quantum numbers of particle physics are interpreted in terms of topological invariants of four-manifolds. The models for electrically charged particles are non-compact four-manifolds which are asymptotically fibred by circles and, for asymptotic U(1) fibrations, the Chern number is identified with minus the electric charge. The asymptotic picture is essentially the electric-magnetic dual of the Kaluza-Klein description of electromagnetism.

In [1], Euclidean Taub-NUT space (TN) is proposed as a geometric model of the electron. TN is more conventionally interpreted as the Kaluza-Klein geometrisation of a magnetic monopole $[8,9]$, and the results of our paper are interesting when viewed from this perspective, too. However, for definiteness we adopt the dual interpretation given in [1] in the following discussion. 
Up to scale, TN is the unique complete hyperkähler four-manifold with isometry group $\mathrm{U}(2)$. With the complex structure defined by the central $\mathrm{U}(1)$ subgroup of $\mathrm{U}(2)$, it is naturally isomorphic to $\mathbb{C}^{2}$. The hyperkähler structure induces a map from TN to Euclidean three-space $\mathbb{E}^{3}$ through its moment map. The moment map intertwines the $\mathrm{SU}(2)$ action on $\mathrm{TN}$ with the $\mathrm{SO}(3)$ action on $\mathbb{E}^{3}$.

The central U(1) action has one fixed point, called the NUT. Away from the NUT, the TN geometry has the structure of a $\mathrm{U}(1)$ bundle, and the hyperkähler moment map is the projection of this fibration. In the interpretation of $\mathrm{TN}$ as a model for the electron, the Chern number of the fibration is the negative of the electric charge and the radius of the fibres over infinity in $\mathbb{E}^{3}$ is the classical electron radius. The model captures the point-like nature of the electron through the unique fixed point of the U(1) action, but does so in the context of a smooth geometry with an associated length scale.

In [1], it was proposed that the spin degrees of elementary particles may be captured in geometric models through the zero-modes of the Dirac operator on the relevant fourmanifold. This was elaborated in [2] where the zero-modes of the Dirac operator on TN minimally coupled to an abelian connection were studied in detail, building on earlier papers by Pope $[10,11]$. Up to an overall factor $p$, the curvature of the abelian connection is the unique harmonic and square integrable two-form on TN. The space of zero modes turns out to combine all irreducible $\mathrm{SU}(2)$ representations up to a dimension determined by $p$ [2].

Here we begin, in section 2 , by revisiting the TN model for the electron, including its spin. The scale parameter in the TN geometry plays a crucial role in our discussion and we explain its relation to the asymptotic size of the U(1) fibres. We emphasise two distinct geometrical aspects of TN space, namely its U(1) fibres, which we interpret as microscopic and having a radius comparable to the classical electron radius, and its three-dimensional base which we interpret as macroscopic and referring to positions in ordinary Euclidean space.

Using the explicit forms of the zero-modes given in [2], we then exhibit a natural link between the spin 1/2 zero-modes of the Dirac operator and complex coordinates on TN defined in terms of the $\mathrm{U}(1)$ fibre. The resulting picture is one where $\mathrm{TN}$ is viewed microscopically as spin space and macroscopically as a U(1)-bundle over the position space $\mathbb{E}^{3}$ of a non-relativistic electron.

Our time-dependent model is introduced and discussed in section 3. It involves a remarkably simple solution of the (4+1)-dimensional Einstein equations first given in [7] which can be interpreted as an evolving TN geometry with the scale parameter changing in time. Surprisingly, allowing the harmonic two-form and the zero-modes of the Dirac operator on TN to change adiabatically with time gives exact solutions of the Maxwell and Dirac equations on the $(4+1)$-dimensional background provided the coefficient $p$ of the abelian connection is kept constant at an integer value. Thus, our interpretation of zero-modes as spin $1 / 2$ states of the electron carries over to the time-dependent setting.

During the time evolution, the microscopic length scale decreases relative to the macroscopic length scale of the TN geometry. As anticipated, our model thereby becomes a natural illustration of Dirac's Large Number Hypothesis [3-6]. This is explained and elaborated in our final section 4 where we discuss the interpretation of the evolving TN geometry as a model for the electron as well as possible generalisations. 


\section{Spin and position in the Taub-NUT geometry}

\subsection{Notation and conventions}

For a more detailed discussion and to fix our conventions we describe the TN geometry in terms of $\mathrm{SU}(2)$-orbits and a transverse coordinate $r$. We parametrise $h \in \mathrm{SU}(2)$ further in terms of a pair of complex numbers $z=\left(z_{1}, z_{2}\right)$ as

$$
h=\left(\begin{array}{rr}
z_{1} & -\bar{z}_{2} \\
z_{2} & \bar{z}_{1}
\end{array}\right), \quad\left|z_{1}\right|^{2}+\left|z_{2}\right|^{2}=1,
$$

and introduce $s u(2)$ generators $t_{j}=-\frac{i}{2} \tau_{j}$, where $\tau_{j}, j=1,2,3$, are the Pauli matrices. Then defining left-invariant one-forms $\sigma_{j}$ via

$$
h^{-1} d h=t_{1} \sigma_{1}+t_{2} \sigma_{2}+t_{3} \sigma_{3},
$$

the TN metric is of the general Bianchi IX form

$$
d s^{2}=f^{2} d r^{2}+a^{2} \sigma_{1}^{2}+b^{2} \sigma_{2}^{2}+c^{2} \sigma_{3}^{2} .
$$

In four dimensions, the hyperkähler property is equivalent to the self-duality of the Riemann tensor with respect to an orientation which is opposite to that defined by the hyperkähler complex structures. As explained in detail in [1], this is in fact the complex structure defined by the central $\mathrm{U}(1)$ subgroup of the isometry group $\mathrm{U}(2)$. In our conventions for the left-invariant one-forms on $\mathrm{SU}(2)$ (which are those of [2] but have the opposite sign of the forms used in [1], so satisfy $\left.d \sigma_{3}=\sigma_{2} \wedge \sigma_{1}\right)$ the orientation is given by the following co-frame

$$
e_{1}=a \sigma_{1}, \quad e_{2}=b \sigma_{2}, \quad e_{3}=c \sigma_{3}, \quad e_{4}=-f d r
$$

and associated volume element

$$
e_{1} \wedge e_{2} \wedge e_{3} \wedge e_{4}=f a b c d r \wedge \sigma_{1} \wedge \sigma_{2} \wedge \sigma_{3}
$$

For a self-dual metric, the coefficient functions satisfy the self-duality equations

$$
\frac{2 b c}{f} \frac{d a}{d r}=(b-c)^{2}-a^{2},+ \text { cycl. }
$$

where '+ cycl.' means we add the two further equations obtained by cyclic permutation of $a, b, c$. For the TN solution, the central U(1) symmetry imposes $a=b$, so the equation for $c$ becomes

$$
\frac{d c}{d r}=-\frac{f c^{2}}{2 a^{2}}
$$

We can fix the coordinate $r$ transverse to the $\mathrm{SU}(2)$ orbits by picking any nowhere vanishing function $f$. There are two geometrically natural choices which are important for us. 


\subsection{Microscopic or spin coordinates}

One natural choice is to pick $f=-2 a^{2} / c^{2}$, so that $r=c$. Geometrically, $2 c$ is the radius of the $\mathrm{U}(1)$ fibres, the factor of two stemming from the angular range $[0,4 \pi)$ of a $U(1)$ subgroup of $\mathrm{SU}(2)$. To write the metric in terms of this coordinate, we divide the equation for $a$ by the equation for $c$ in (2.6) to find

$$
\frac{d a}{d c}=-\frac{a}{c}+2\left(\frac{a}{c}\right)^{2} .
$$

The TN solution has $a=0$ when $c=0$, but this does not fix the solution of (2.8) since $c=0$ is a singular point. Instead, the general solution is

$$
a=\frac{c}{1-\frac{c^{2}}{\Lambda^{2}}}
$$

and involves an arbitrary positive constant $\Lambda$ which fixes the range of $c$ as $[0, \Lambda)$ and the asymptotic radius of the $\mathrm{U}(1)$ fibres as $2 \Lambda$. There is, therefore, a one-parameter family of TN metrics, labelled by the size of the asymptotic circle. The metric depends on $\Lambda$ and takes the form

$$
d s^{2}=\frac{4}{\left(1-\frac{c^{2}}{\Lambda^{2}}\right)^{4}} d c^{2}+\frac{c^{2}}{\left(1-\frac{c^{2}}{\Lambda^{2}}\right)^{2}}\left(\sigma_{1}^{2}+\sigma_{2}^{2}\right)+c^{2} \sigma_{3}^{2} .
$$

There is a family of 'cigar shaped' geodesic submanifolds which are invariant under the global $\mathrm{U}(1)$ action, parametrised by the coset $\mathrm{SU}(2) / \mathrm{U}(1)$ which, as we shall see, is the two-sphere of spatial directions in the base $\mathbb{E}^{3}$. The metric on each submanifold is

$$
d s^{2}=\frac{4}{\left(1-\frac{c^{2}}{\Lambda^{2}}\right)^{4}} d c^{2}+c^{2} d \gamma^{2}
$$

where $\gamma$ is an angular coordinate with range $[0,4 \pi)$. The Gauss curvature at the tip of the cigar (the NUT in TN) is

$$
K=\frac{1}{\Lambda^{2}}
$$

The curvature radius at the NUT is half the asymptotic radius of the fibres, showing that the asymptotic scale $\Lambda$ can also be read off from the geometry near the NUT.

For later use we note that, with $c$ as coordinate, the proper radial distance is

$$
\rho(c)=\int_{0}^{c} \frac{2 d s}{\left(1-\frac{s^{2}}{\Lambda^{2}}\right)^{2}}=\frac{c}{\left(1-\frac{c^{2}}{\Lambda^{2}}\right)}+\Lambda \tanh ^{-1}\left(\frac{c}{\Lambda}\right),
$$

and that this relation is linear $\rho(c) \approx 2 c$ for small $c$.

We can use the fibre radius $2 c$ to define global complex coordinates on $\mathrm{TN}$ via

$$
w=2 c z .
$$

Then $w=\left(w_{1}, w_{2}\right)$ takes values in the open ball $B_{\Lambda}=\left\{w \in \mathbb{C}^{2}|| w \mid<2 \Lambda\right\}$ of radius $2 \Lambda$. We refer to $w$ as a microscopic coordinate since its magnitude is related to the fibre radius 
whose asymptotic value $\Lambda$ was interpreted as the classical electron radius in [1]. Since $\frac{1}{4}\left(\sigma_{1}^{2}+\right.$ $\left.\sigma_{2}^{2}+\sigma_{3}^{2}\right)$ is the round metric on the unit three-sphere, the TN metric near the NUT is flat:

$$
d s^{2} \approx\left|d w_{1}\right|^{2}+\left|d w_{2}\right|^{2} .
$$

As we shall explain in section 2.4, the coordinates $w_{1}$ and $w_{2}$ are naturally interpreted as spin coordinates for TN. They obviously transform in the fundamental representation of $\mathrm{SU}(2)$, but more specifically we shall show that the spin $1 / 2$ zero-modes of a twisted Dirac operator on TN are linear functions of them.

\subsection{Macroscopic or position coordinates}

A second, geometrically natural choice of radial coordinate is provided by the $\mathrm{U}(1)$ fibration of TN over $\mathbb{E}^{3}$ away from the origin. For any fixed $\mathrm{SU}(2)$ orbit, this is the Hopf fibration

$$
\pi: S^{3} \rightarrow S^{2}, \quad z \mapsto \vec{n}=z^{\dagger} \vec{\tau} z .
$$

Using the Euler angle parametrisation of SU(2) given in [2],

$$
z_{1}=e^{-\frac{i}{2}(\alpha+\gamma)} \cos \frac{\beta}{2}, \quad z_{2}=e^{\frac{i}{2}(\alpha-\gamma)} \sin \frac{\beta}{2}, \quad \beta \in[0, \pi), \alpha \in[0,2 \pi), \gamma \in[0,4 \pi),
$$

we have $\vec{n}=(\sin \beta \cos \alpha, \sin \beta \sin \alpha, \cos \beta)$ and so

$$
\sigma_{1}^{2}+\sigma_{2}^{2}=d \beta^{2}+\sin ^{2} \beta d \alpha^{2}
$$

is the standard round metric on the unit two-sphere parametrised by $\vec{n}$. The choice

$$
f=-\frac{a}{r},
$$

then makes the metric isotropic in the coordinate vector

$$
\vec{x}=r \vec{n},
$$

i.e., it brings it into the form

$$
d s^{2}=\frac{a^{2}}{r^{2}} d \vec{x}^{2}+c^{2} \sigma_{3}^{2} .
$$

Observe that $r$, like $a$ and $c$, necessarily has the dimension length. To determine $a$ as a function of $r$ we substitute (2.9) into (2.7) and integrate:

$$
\frac{d}{d r}\left(\frac{r}{c^{2}}\right)=\frac{1}{\Lambda^{2}} \Leftrightarrow \frac{1}{c^{2}}=\frac{1}{\Lambda^{2}}+\frac{1}{r L} .
$$

The solution requires an integration constant of dimension inverse length which we have denoted by $1 / L$. Thus, defining the dimensionless quantitity

$$
\epsilon=\frac{L^{2}}{\Lambda^{2}}
$$

and introducing

$$
V=\epsilon+\frac{L}{r},
$$


we have, from (2.9),

$$
c=\frac{L}{\sqrt{V}}, \quad a=r \sqrt{V},
$$

with the radial coordinate $r$ taking values in $[0, \infty)$.

The metric (2.21) with the expressions (2.25) for $a$ and $c$ is the TN metric

$$
d s^{2}=\left(\epsilon+\frac{L}{r}\right) d \vec{x}^{2}+\frac{r L^{2}}{\epsilon r+L} \sigma_{3}^{2}
$$

in Gibbons-Hawking form. We have re-derived it here to clarify the origin and the interpretation of the two constants $\epsilon$ and $L$ which appear in it. The length scale $\Lambda>0$ fixes the asymptotic radius of the $\mathrm{U}(1)$ fibres. The second length scale $L$ appears when we introduce coordinates on the base $\mathbb{E}^{3}$ of the fibration. For the regular ('positive mass') TN geometry one needs $L>0$ and we assume this in the following. The dimensionless quantity $\sqrt{\epsilon}$ is the ratio of these length scales. Only the scale $\Lambda$ of the fibre has an invariant meaning. This can also be seen by expressing the TN metric (2.26) in terms of the TN metric with $\epsilon=L=1$ via a change of coordinates $\tilde{r}=(\epsilon r) / L$ on the base and an overall re-scaling by $\Lambda^{2}$ :

$$
d s^{2}=\frac{L^{2}}{\epsilon}\left[\left(1+\frac{1}{\tilde{r}}\right) d \tilde{\vec{x}}^{2}+\frac{\tilde{r}}{\tilde{r}+1} \sigma_{3}^{2}\right] .
$$

Defining $R / 2=\sqrt{L r}$ as the geometric mean of $r$ and the scale parameter $L$, so that

$$
r=\frac{1}{4 L} R^{2},
$$

we introduce macroscopic complex coordinates on $\mathrm{TN}$ via

$$
W=R z \in \mathbb{C}^{2} .
$$

They project to $\vec{x}$ via the Hopf projection

$$
\vec{x}=\frac{1}{4 L} W^{\dagger} \vec{\tau} W
$$

Observe that for small $r$, from (2.22) and (2.28),

$$
r \approx \frac{1}{L} c^{2}, \quad R \approx 2 c, \quad w \approx W
$$

so that microscopic and macroscopic coordinates agree near the NUT.

As advertised in the Introduction, the coordinate vector $\vec{x}$ for the base space can be obtained more invariantly from the hyperkähler structures of TN. The three hyperkähler forms $\omega_{i}, i=1,2,3$, are invariant under the $\mathrm{U}(1)$ action. Writing

$$
X=\frac{\partial}{\partial \gamma}
$$

for the vector field generating the $\mathrm{U}(1)$ action, we have $\mathcal{L}_{X} \omega_{i}=0$, so that the forms $\iota_{X} \omega_{i}$ are closed. For the TN metric in the form (2.26), the hyperkähler forms are

$$
\omega_{i}=L d x_{i} \wedge \sigma_{3}+\frac{1}{2} V \epsilon_{i j k} d x_{j} \wedge d x_{k}, \quad i=1,2,3 .
$$


Note that the orientation associated with the hyperkähler structures

$$
\omega_{1} \wedge \omega_{1}=\omega_{2} \wedge \omega_{2}=\omega_{3} \wedge \omega_{3}=L V d x_{1} \wedge d x_{2} \wedge d x_{3} \wedge \sigma_{3}
$$

is the opposite of our orientation (2.5) which is determined by the global U(1) action, as expected. Defining associated moment maps $\mu_{i}$ via

$$
\iota_{X} \omega_{i}=-d \mu_{i},
$$

we find that they are, up to the scale $L$ and additive constants $q_{i}$, the Euclidean position coordinates:

$$
\mu_{i}=L x_{i}+q_{i}, \quad i=1,2,3
$$

\subsection{The twisted Dirac operator on TN space}

Even though TN is topologically trivial, it has non-trivial $L^{2}$-cohomology in the middle dimension [10]. The harmonic representative, unique up to scale, is the exterior derivative of the one-form $c^{2} \sigma_{3}$ (which is not square-integrable). Here we want to interpret the harmonic representative as the curvature of an abelian connection on TN. We adapt the conventions of [2] (where $\epsilon=1$ ) and write the connection one-form as

$$
A=\frac{i p}{2} \frac{c^{2}}{\Lambda^{2}} \sigma_{3}=\frac{i p}{2} \frac{\epsilon r}{\epsilon r+L} \sigma_{3}
$$

so that the curvature is

$$
F=d A=\frac{i \epsilon p}{2}\left(\frac{L}{(\epsilon r+L)^{2}} d r \wedge \sigma_{3}+\frac{r}{\epsilon r+L} \sigma_{2} \wedge \sigma_{1}\right) .
$$

The coefficient $p$ can take arbitrary real values since TN has no non-trivial two-cycles. However, TN can be compactified to $\mathbb{C P}^{2}$ by adding a $\mathbb{C P}^{1}$ representing 'spatial infinity' [1]. In our conventions, this is the $\mathbb{C P}^{1}$ with homogeneous coordinate $w$. If one requires that $A$ extends to a connection on $\mathbb{C P}^{2}$ then $p$ has to take integer values, but we will not assume this in the following.

The index of the Dirac operator on TN minimally coupled to the connection $A$ was computed by Pope in $[10,11]$ as $\frac{1}{2}[|p|]([|p|]+1)$, where $[x]$ is the largest integer strictly smaller than the positive real number $x$. This index and the properties of Dirac zeromodes are studied in more detail in the paper [2] the notation of which we use here. The Dirac operator has the form

$$
\not D_{p}=\left(\begin{array}{cc}
0 & T_{p}^{\dagger} \\
T_{p} & 0
\end{array}\right),
$$

and all its normalisable zero-modes come from normalisable zero-modes of $T_{p}$. As explained in [2], the space of all zero-modes decomposes into the sum of irreducible representations of $\mathrm{SU}(2)$ with spins $j$ satisfying $2 j+1<|p|$. 
Fixing $p>0$ for definiteness, the zero-modes depend holomorphically on the complex coordinates. For fixed $j$, they can be written as

$$
\Psi_{j}\left(r, z_{1}, z_{2}\right)=\left(\begin{array}{c}
R_{j}(r) \sum_{m=-j}^{j} a_{m} z_{1}^{j-m} z_{2}^{j+m} \\
0 \\
0 \\
0
\end{array}\right),
$$

where $a_{m}, m=-j,-j+1, \ldots, j-1, j$, are arbitrary complex constants. The radial dependence is determined by the differential equation

$$
\left(L \partial_{r}+\frac{\epsilon}{2}(p-(2 j+1))+\left(\frac{1}{2}-j\right) \frac{L}{r}-\frac{L^{2}}{2 r(\epsilon r+L)}\right) R_{j}(r)=0
$$

which has the general solution

$$
R_{j}(r)=C \frac{r^{j}}{\sqrt{\epsilon r+L}} e^{((2 j+1)-p) \frac{\epsilon r}{2 L}}
$$

Here $C$ is a normalisation constant and $j$ is the spin, required to satisfy $(2 j+1)<p$ for normalisability.

In [2], the $j=1 / 2$ doublet of states was proposed as a model for the spin degrees in the TN model. It was also pointed out that, with the choice $p=2$, the spin $1 / 2$ states are not square-integrable but have the form of a vortex, with constant magnitude at spatial infinity. Remarkably, the radial dependence is a multiple of the fibre radius $2 c(2.25)$ in the TN metric. As a result, the first component of the spinor in (2.40) is simply a linear function in the global coordinate $w(2.14)$ on $\mathrm{TN}$, so that we can write the zero-modes as

$$
\Psi_{\frac{1}{2}}\left(r, z_{1}, z_{2}\right)=\left(\begin{array}{c}
a_{-\frac{1}{2}} w_{1}+a_{\frac{1}{2}} w_{2} \\
0 \\
0 \\
0
\end{array}\right) .
$$

This justifies our earlier interpretation of $w_{1}$ and $w_{2}$ as coordinates on spin space.

\subsection{Scaling properties and the Landau limit}

In the limit $\epsilon \rightarrow 0$, the $\mathrm{U}(1)$ fibre decompactifies and $\mathrm{TN}$ space becomes flat $\mathbb{R}^{4}$. The microscopic and macroscopic coordinates $w$ and $W$ now coincide since, when $\epsilon=0$,

$$
c=\frac{R}{2}=\sqrt{L r} .
$$

The TN metric is flat and given by $\left|d W_{1}\right|^{2}+\left|d W_{2}\right|^{2}$ in this limit. For negative values of $\epsilon$, the metric coefficients (2.25) still satisfy the self-duality equations but the metric (2.26) is now minus the 'negative mass' TN geometry, which is singular at $r=-L / \epsilon$. This metric arises as the asymptotic form of the Atiyah-Hitchin metric on the monopole moduli space [12]. 
With our normalisation, the abelian connection (2.37) and curvature (2.38) vanish in the limit $\epsilon \rightarrow 0$, but it is instructive to consider the limit

$$
\epsilon \rightarrow 0, \quad \epsilon p \rightarrow \tilde{p} \neq 0
$$

The connection becomes

$$
A=i \frac{\tilde{p} R^{2}}{8 L^{2}} \sigma_{3}=\frac{\tilde{p}}{8 L^{2}}\left(W_{1} d \bar{W}_{1}+W_{2} d \bar{W}_{2}-\bar{W}_{1} d W_{1}-\bar{W}_{2} d W_{2}\right)
$$

so that the curvature is essentially the Kähler form on $\mathbb{C}^{2}$ :

$$
F=\frac{\tilde{p}}{4 L^{2}}\left(d W_{1} \wedge d \bar{W}_{1}+d W_{2} \wedge \bar{W}_{2}\right) .
$$

Physically, this can be thought of as a constant magnetic field in both the $W_{1}$ and the $W_{2}$ plane. It is not surprising, therefore, that the Dirac zero-modes become products of Landau ground states in each of the planes, with a Gaussian radial function multiplying holomorphic polynomials in $W_{1}$ and $W_{2}$ :

$$
W_{1}^{j-m} W_{2}^{j+m} e^{-\tilde{p} \frac{|W|^{2}}{2 L}}
$$

This is the Landau limit of our model. All energy levels, including the zero-energy state, have an infinite degeneracy in this limit.

\section{Introducing time}

Somewhat surprisingly, an affine time dependence of the scaling parameter $\epsilon$ gives rise to an exact solution of the (4+1)-dimensional vacuum Einstein equations. This solution was first obtained in [7] as the Kaluza-Klein form of the electric-magnetic dual of a KastorTraschen solution of the Einstein-Maxwell equations. For our purposes, it is instructive to derive it directly as follows.

We write $g_{\mathrm{T} N}(t)$ for the TN metric (2.26) with the parameter $\epsilon$ depending on an additional time variable $t$, i.e., we consider the adiabatic variation of the potential

$$
V=\epsilon(t)+\frac{L}{r}
$$

Then the metric

$$
d s^{2}=-d t^{2}+g_{\mathrm{TN}}(t)
$$

has Ricci scalar

$$
s=\frac{2 r}{\epsilon(t) r+L} \ddot{\epsilon}
$$

with dots denoting derivatives with respect to $t$. Moreover, in terms of the coefficient function (2.25) and the co-frame

$$
e^{0}=d t, \quad e^{1}=a \sigma_{1}, \quad e^{2}=a \sigma_{2}, \quad e^{3}=c \sigma_{3}, \quad e^{4}=\frac{a}{r} d r
$$


the Ricci tensor has non-vanishing components

$$
\operatorname{Ric}_{00}=-\frac{1}{2} s, \quad \operatorname{Ric}_{11}=\operatorname{Ric}_{22}=-\operatorname{Ric}_{33}=\operatorname{Ric}_{44}=\frac{1}{4} s .
$$

Thus

$$
\operatorname{Ric}=0 \Leftrightarrow \ddot{\epsilon}=0,
$$

so that an affine dependence of $\epsilon$ on $t$ yields a Ricci-flat metric. Obtaining an exact solution of a time-dependent problem from an adiabatic ansatz is rather unusual and it is not clear to us why it works in this instance.

Concentrating on the simplest case

$$
\epsilon(t)=t
$$

the family of metrics $g_{\mathrm{T} N}(t)$ starts, at $t=0$, with Euclidean four-space. For $t>0$ one dimension compactifies to the circle fibre of $\mathrm{TN}$ space, with the radius of the circle decreasing with $t$ as

$$
\Lambda(t)=\frac{L}{\sqrt{t}} .
$$

For $t<0$ the metric $g_{\mathrm{TN}}(t)$ is the negative of the (singular) negative mass TN metric, so that (3.2) is a five-dimensional singular space whose signature flips from $(-,+,+,+,+)$ to $(-,-,-,-,-)$, with the two regimes separated by the singular region $r t=-L$.

In order to extend our description of spin to the time-dependent case we look for solutions of the Maxwell and Dirac equations on the (4+1)-dimensional space-time (3.2). We continue to work with the co-frame (2.4), use Greek indices $\mu, \nu, \ldots$ in the range $0, \ldots, 4$ and raise or lower them with the 'mostly plus' metric $\operatorname{diag}(-1,1,1,1,1)$. It is then straightforward to check that allowing $\epsilon$ and $p$ to vary with time, the one-form

$$
A=\frac{i p(t)}{2} \frac{\epsilon(t) r}{\epsilon(t) r+L} \sigma_{3}
$$

gives rise to the curvature

$$
F=d A=\frac{i}{2}\left(\frac{(\dot{\epsilon} p-\dot{p} \epsilon) r L-\dot{p} L^{2}}{(\epsilon r+L)^{2}} d t \wedge \sigma_{3}+\frac{p \epsilon L}{(\epsilon r+L)^{2}} d r \wedge \sigma_{3}+\frac{p \epsilon r}{\epsilon r+L} \sigma_{2} \wedge \sigma_{1}\right),
$$

which satisfies

$$
d \star F=0,
$$

provided that

$$
\ddot{\epsilon}=0 \quad \text { and } \quad \ddot{p}=0 .
$$

In other words, evolving the abelian gauge field $A$ adiabatically with the TN metric gives a solution of the Maxwell equations. However, the energy-momentum tensor does not vanish, so we do not obtain a solution of the coupled Einstein-Maxwell equations.

For the Dirac equation in $4+1$ dimensions we use the gamma matrices

$$
\gamma_{0}=\left(\begin{array}{cc}
\mathbf{1}_{2} & 0 \\
0 & -\mathbf{1}_{2}
\end{array}\right), \quad \gamma_{i}=\left(\begin{array}{cc}
0 & \tau_{j} \\
-\tau_{j} & 0
\end{array}\right), \quad \gamma_{4}=\left(\begin{array}{cc}
0 & -i \mathbf{1}_{2} \\
-i \mathbf{1}_{2} & 0
\end{array}\right)
$$


The spin connection one-form for (3.2), defined via

$$
d e^{\mu}+\omega^{\mu}{ }_{\nu} \wedge e^{\nu}=0
$$

has the four-dimensional Euclidean components given, for example, in [2] and the additional components

$$
\omega_{01}=-\frac{\dot{\epsilon} r}{2 \sqrt{\epsilon+\frac{L}{r}}} \sigma_{1}, \omega_{02}=-\frac{\dot{\epsilon} r}{2 \sqrt{\epsilon+\frac{L}{r}}} \sigma_{2}, \omega_{03}=\frac{\dot{\epsilon} L}{2\left(\epsilon+\frac{L}{r}\right)^{\frac{3}{2}}} \sigma_{3}, \omega_{04}=-\frac{\dot{\epsilon}}{2 \sqrt{\epsilon+\frac{L}{r}}} d r .
$$

In terms of the left-invariant vector fields $X_{1}, X_{2}, X_{3}$ on $\mathrm{SU}(2)$ which are dual to the one-forms $\sigma_{1}, \sigma_{2}, \sigma_{3}$, the frame field dual to the co-frame (2.4) is

$$
E_{0}=\frac{\partial}{\partial t}, \quad E_{1}=\frac{1}{a} X_{1}, \quad E_{2}=\frac{1}{a} X_{2} \quad E_{3}=\frac{1}{c} X_{3}, \quad E_{4}=\frac{r}{a} \frac{\partial}{\partial r} .
$$

Then, with $A$ standing for the $t$-dependent one-form (2.37) and

$$
A_{\mu}=A\left(E_{\mu}\right), \quad \omega_{\mu}^{\kappa \lambda}=\omega^{\kappa \lambda}\left(E_{\mu}\right)
$$

the Dirac operator is

$$
\not D_{p, t}=\gamma^{\mu}\left(E_{\mu}+A_{\mu}-\frac{1}{8}\left[\gamma_{\kappa}, \gamma_{\lambda}\right] \omega_{\mu}^{\kappa \lambda}\right) .
$$

Inserting (2.25) for $a$ and $c$ and the spin connection, this simplifies to

$$
\not D_{p, t}=\gamma^{0}\left(\frac{\partial}{\partial t}+\frac{1}{2} \frac{\dot{\epsilon} r}{\epsilon r+L}\right)+\not D_{p}
$$

where $\not_{p}$ is the four-dimensional Dirac operator (2.39) for the metric $g_{\mathrm{T} N}(t)$, coupled to the connection (3.9) at some given $t$.

Rather remarkably, we can obtain solutions of the time-dependent Dirac equation

$$
\not D_{p, t} \Psi=0
$$

by taking zero-modes of the form (2.40) with adiabatically varying radial dependence

$$
R_{j}(r, t)=C \frac{r^{j}}{\sqrt{\epsilon(t) r+L}} e^{((2 j+1)-p(t)) \frac{\epsilon(t) r}{2 L}}
$$

provided that

$$
\frac{\partial}{\partial t} R_{j}(r, t)=-\frac{1}{2} \frac{\dot{\epsilon} r}{\epsilon r+L} R_{j}(r, t)
$$

This holds provided $p$ is constant and satisfies

$$
p=2 j+1,
$$

so that the exponential factor in (3.21) vanishes. 
Thus, we recover the quantisation condition on $p$ which ensures that for fixed $t$ the connection (3.9) extends to $\mathbb{C P}^{2}$ as the condition for zero-energy solutions of the timedependent Dirac equation (3.20). The resulting solutions have the radial dependence

$$
R_{j}(r, t)=C \frac{r^{j}}{\sqrt{\epsilon(t) r+L}},
$$

and are not square-integrable over TN for any fixed value of $t$.

Again, the $j=1 / 2$ solution is special in that it tends to a constant at spatial infinity and is proportional to the fibre radius $2 c$ for any value of $t$. Extending the earlier definitions to hold for any fixed $t$

$$
c(t, r)=\frac{L}{\sqrt{\epsilon(t)+\frac{L}{r}}}, \quad w(t, r, z)=c(t, r) z,
$$

the formula (2.43) for the spin $1 / 2$ solution in terms of $w$ also holds in the time-dependent case:

$$
\Psi_{\frac{1}{2}}\left(t, r, z_{1}, z_{2}\right)=\left(\begin{array}{c}
a_{-\frac{1}{2}} w_{1}+a_{\frac{1}{2}} w_{2} \\
0 \\
0 \\
0
\end{array}\right) .
$$

The energy-momentum tensor for the spinor (3.26) with radial and timedependence (3.24) does not vanish. The metric (3.2), the gauge potential (3.9) and the spinor $(3.26)$ with $(r, t)$-dependence (3.24) satisfy, respectively, the Einstein equations (provided $\epsilon$ is affine in $t$ ), the Maxwell equations (provided, in addition, that $p$ is affine in $t$ ) and the Dirac equation (provided, in addition, that $p$ is constant and an integer); they do not satisfy the coupled Einstein-Maxwell-Dirac system.

\section{Geometric models of particles and Dirac's Large Number Hypothesis}

The idea of describing particles in terms of everywhere smooth solutions of the Einstein equations was explored by Einstein and Rosen almost 80 years ago in [13]. Implementing this idea in a realistic model of elementary particles is beset with various well-known difficulties, the most basic of which is the problem of scales.

The problem is illustrated by a close cousin of the model discussed in in this paper, namely the trivial (4+1)-dimensional extension of the static Taub-NUT geometry into a solution of the Einstein equations. In [8, 9], this spacetime is interpreted as a static KaluzaKlein monopole, and it is shown that the mass of the monopole is proportional to the ratio of the asymptotic fibre radius to Newton's constant in $3+1$ dimensions. As a result, the mass associated to the typical length scale $10^{-15} \mathrm{~m}$ of nuclear physics is a huge $10^{12} \mathrm{~kg}$, equivalent to the mass of $10^{39}$ protons.

The time-dependent geometric model of a particle discussed in this paper continues the line of thought started by Einstein and Rosen but needs to be interpreted with care in order to avoid unrealistic relations between size and mass of the kind described above. 
As in the discussion of static models in [1], we do this in the first instance by avoiding the problem of unification and aiming at a geometrisation of particles and their nongravitational interactions only. Also note that the models in [1] and the time-dependent extension introduced here do not yet include a theory of energy and dynamics, though proposals for both have been put forward [14].

The TN model of the electron as proposed in [1] only captures basic properties of the electron: the negative of the electron charge $e$ through the Chern class of the asymptotic $\mathrm{U}(1)$ fibration, the point-like nature of the electron through the single fixed-point of the $\mathrm{U}(1)$ action (the NUT), and the mass of the electron through the interpretation of the asymptotic length $\Lambda$ of the $\mathrm{U}(1)$ fibre as the classical electron radius. The electron mass, in units where the speed of light is one, is therefore

$$
m_{e}=\frac{e^{2}}{\Lambda}
$$

An additional insight gained in the current paper is that TN space is naturally interpreted as the spin space of the electron in the sense that spin $1 / 2$ zero-modes of the twisted Dirac operator are linear functions of the microscopic complex coordinate (2.14) defined in terms of the $\mathrm{U}(1)$ action and its orbit length. Thus the beautiful link between spin $1 / 2$ and the unit charge Dirac monopole via the Hopf fibration finds a natural place in the TN model of the electron.

Having arrived at this interpretation through the study of conventional spinors obeying the Dirac equation on TN, it is tempting to discard the Dirac operator and to simply postulate that spin $1 / 2$ states of the electron are the linear functions of the microscopic complex TN coordinates. Either way, the resulting picture is one where the TN geometry has two facets, one microscopic and referring to electron's spin degrees of freedom, and the other macroscopic and referring to its position in Euclidean three-space.

Put this way, the picture, already incorporating Dirac's monopole and equation, becomes an illustration of yet another of Dirac's ideas, namely his proposal of two metrics, made in the context of his Large Number Hypothesis (LNH).

Briefly, the LNH states that large numbers in physics such as the ratio of the electromagnetic to gravitational force between proton and electron are of the same order of magnitude as the age of the universe in atomic units [3-6]. This necessarily requires one or more of the fundamental constants of nature to vary with the age of the universe. Dirac proposed that Newton's constant $G$ should vary while the masses of elementary particles should remain constant.

Since Einstein's theory of gravity requires a constant value for $G$, Dirac then postulated two systems of units, Einstein units in which Einstein's equations hold, and atomic units in which $G$ varies with time and Einstein's equations do not necessarily hold. There are two distinct metrics, one for each set of units.

It turns out that our microscopic and macroscopic interpretation of the TN geometry are illustrations of Dirac's LNH and two-metric formalism. In fact, several aspects of the TN model may be viewed as precise versions of ideas proposed by Dirac in the context of the LNH, as we shall now explain. 
Far from the NUT, i.e., for large $r$, the $\mathrm{U}(1)$ fibre is negligible and the metric is essentially the flat metric $d \vec{x}^{2}$ of macroscopic Euclidean space. Near the NUT, i.e., for small $r$, the metric is the flat metric (2.15) on microscopic spin space. The relation between the macroscopic line element $d r$ and the microscopic line element $d c$ for small $r$ is, according to $(2.31)$,

$$
L d r \approx c d c .
$$

This relation is nothing but the spatial version of Dirac's formula, derived using dimensional analysis in [6],

$$
d \tau=t d t
$$

which relates the time $\tau$ measured in Einstein units and the time $t$ measured in atomic units. It is this similarity which inspired the current paper.

Dirac argued that the masses of particles should be the same whether they are expressed in atomic or Einstein units. In our model, this is captured by the fact that the length scale $\Lambda$ appearing in the mass formula (4.1) is defined in terms of the asymptotic geometry but can also be extracted from the geometry at the NUT according to (2.12). This is a non-trivial feature of the TN geometry which implies the equality of 'local' and 'asymptotic' mass.

Finally, the time-dependent solution (3.2) with affine evolution of $\epsilon$ captures yet further aspects of the LNH. As we saw, the metric satisfies the $(4+1)$-dimensional Einstein equations and the abelian gauge potential and spinor satisfy the linear Maxwell and Dirac equations, but they do not satisfy the non-linear and fully coupled Einstein-Maxwell-Dirac equations. From Dirac's point of view, this is not surprising since the coupling between matter and geometry in atomic units needs not be of the usual Einstein form.

The details of the time evolution of length scales in the time-dependent geometry (3.2) also fit well with the LNH. Recall from $(2.23)$ that $\sqrt{\epsilon}$ is the ratio of two length scales, namely the macroscopic length scale $L$ for the base of TN and the microscopic length scale $\Lambda$ of the fibre. Concentrating again on the case $\epsilon(t)=t$, the resulting metric

$$
d s^{2}=\left(t+\frac{L}{r}\right) d \vec{x}^{2}+\frac{L^{2} r}{t r+L} \sigma_{3}^{2}
$$

has the asymptotic (large $r$ ) form

$$
d s^{2} \approx t d \vec{x}^{2}+\frac{L^{2}}{t} \sigma_{3}^{2} .
$$

To compare length scales in the base and the fibre, we introduce dimensionless spatial coordinates $\vec{\xi}=\vec{x} / L$, matching the dimensionless angular coordinate $\gamma \in[0,4 \pi)$ on the fibre. The metric then reads

$$
d s^{2} \approx t L^{2} d \vec{\xi}^{2}+\frac{L^{2}}{t} \sigma_{3}^{2} .
$$

The macroscopic length scale $L_{M}$ for the base and the microscopic length scale $L_{m}$ for the fibre are thus

$$
L_{M}=\sqrt{t} L, \quad L_{m}=\frac{L}{\sqrt{t}},
$$


and so

$$
\frac{L_{M}}{L_{m}}=t .
$$

Identifying the order of magnitude of $L_{M}$ with the length scale associated to the current estimate $10^{-52} \mathrm{~m}^{-2}$ for the cosmological constant,

$$
L_{M}=10^{26} \mathrm{~m},
$$

and the order of magnitude of $L_{m}$ with the classical electron radius

$$
L_{m}=\frac{e^{2}}{m_{e} c^{2}} \approx 10^{-15} \mathrm{~m},
$$

we indeed find that $L_{M} / L_{m} \approx 10^{41}$ is close to Dirac's large number. This suggests the interpretation of $t$ as the age of the universe in atomic units and thus the following tentative interpretation of the model as a geometric description of the electron's time evolution since the big bang.

Identifying $t=0$ with the time of the big bang, the initial geometry is $\mathbb{R}^{4}$, but for $t>0$ one dimension compactifies and becomes the fibre in a dual Kaluza-Klein model of electromagnetism. The size of the internal dimension is initially comparable to macroscopic scales, but it shrinks as time evolves. From this point of view, the reason why the classical electron radius is small relative to cosmological length scales today is simply that this shrinking has gone on for a long time. Note also that this model of a single electron comes with a preferred direction of time. As discussed after (3.8), backward evolution from $t=0$ leads to a singular spacetime while forward evolution is smooth.

To sum up, we developed the description of spin in a geometric model of a particle and proposed a precise way of including time. We showed that the description of spin can naturally be extended to the time-dependent model and that the resulting picture captures several key aspects of Dirac's LNH in a mathematically precise way.

A model of a single particle necessarily only has limited scope for phenomenology and it is therefore important to extend the ideas of this paper to multi-particle models. MultiTN space is a natural arena for modelling multi-electron systems and it was shown in [7] that the time evolution discussed here for $\mathrm{TN}$ can be extended to the multi-TN metric. The interacting dynamics of electrons and their spins could probably be discussed by exploiting and combining the index results in [10] with the proposals for interaction energies in [14], but the details have yet to be worked out.

One would also like to extend both the description of spin and the inclusion of time to models of baryons. In [1], the Atiyah-Hitchin ( $\mathrm{AH}$ ) metric was proposed as a (static) model for the proton. The AH model is rotationally symmetric but only has an additional $\mathrm{U}(1)$ isometry asymptotically. It captures the extended nature of the proton through a 'core' region where the $\mathrm{U}(1)$ action is not defined.

In light of the essential role played by the $\mathrm{U}(1)$ fibration in this paper, it may be more natural to consider only Ricci-flat four-manifolds having a globally defined U(1) action as models for static particles. The Gibbons-Hawking classification of the possible fixed point sets as either isolated 'nuts' or two-dimensional 'bolts' [15] suggests that the former could describe electrons and the latter baryons. The Euclidean Schwarzschild metric and the Taub-bolt (or Page) metric [16] would then be natural candidates for the neutron and the 
proton. It is therefore interesting to find time-dependent versions of these models, ideally also including a description of spin, and to interpret them along the lines of this paper.

\section{Acknowledgments}

We thank José Figueroa O'Farrill for numerous discussions and acknowledge support from the EPSRC through a research grant. BJS also thanks Maciej Dunajski for discussions.

Open Access. This article is distributed under the terms of the Creative Commons Attribution License (CC-BY 4.0), which permits any use, distribution and reproduction in any medium, provided the original author(s) and source are credited.

\section{References}

[1] M. Atiyah, N.S. Manton and B.J. Schroers, Geometric models of matter, Proc. Roy. Soc. Lond. A 468 (2012) 1252 [arXiv:1108.5151] [InSPIRE].

[2] R. Jante and B.J. Schroers, Dirac operators on the Taub-NUT space, monopoles and $\mathrm{SU}(2)$ representations, JHEP 01 (2014) 114 [arXiv:1312.4879] [INSPIRE].

[3] P.A.M. Dirac, New basis for cosmology, Proc. Roy. Soc. Lond. A 165 (1938) 199 [inSPIRE].

[4] P.A.M. Dirac, Long range forces and broken symmetries, Proc. Roy. Soc. Lond. A 333 (1973) 403 [INSPIRE].

[5] P.A.M. Dirac, Cosmological models and the large numbers hypothesis, Proc. Roy. Soc. A 338 (1974) 439.

[6] P.A.M. Dirac, The large numbers hypothesis and the Einstein theory of gravitation, Proc. Roy. Soc. Lond. A 365 (1979) 19 [INSPIRE].

[7] G.W. Gibbons, H. Lü and C.N. Pope, Brane worlds in collision, Phys. Rev. Lett. 94 (2005) 131602 [hep-th/0501117] [INSPIRE].

[8] R. Sorkin, Kaluza-Klein monopole, Phys. Rev. Lett. 51 (1983) 87 [inSPIRE].

[9] D.J. Gross and M.J. Perry, Magnetic monopoles in Kaluza-Klein theories, Nucl. Phys. B 226 (1983) 29 [inSPIRE].

[10] C.N. Pope, Axial vector anomalies and the index theorem in charged Schwarzschild and Taub-NUT spaces, Nucl. Phys. B 141 (1978) 432 [INSPIRE].

[11] C.N. Pope, The $\eta$ invariant for charged spinors in Taub-NUT, J. Phys. A 14 (1981) L133 [INSPIRE].

[12] G.W. Gibbons and N.S. Manton, Classical and quantum dynamics of BPS monopoles, Nucl. Phys. B 274 (1986) 183 [inSPIRE].

[13] A. Einstein and N. Rosen, The particle problem in the general theory of relativity, Phys. Rev. 48 (1935) 73 [INSPIRE].

[14] G. Franchetti and N.S. Manton, Gravitational instantons as models for charged particle systems, JHEP 03 (2013) 072 [arXiv:1301.1624] [INSPIRE].

[15] G.W. Gibbons and S.W. Hawking, Classification of gravitational instanton symmetries, Commun. Math. Phys. 66 (1979) 291 [INSPIRE].

[16] D.N. Page, Taub-NUT instanton with an horizon, Phys. Lett. B 78 (1978) 249 [inSPIRE]. 